\title{
COLLEGE NEWS AND CPD PROGRAMME
}

(J Bangladesh Coll Phys Surg 2011; 29: 247-253)

Examinations news:

Results of FCPS Part-I, Part-II and MCPS examination held in July 2011 are given bellow:

3813 candidates appeared in FCPS Part-I examination held in July, 2011 of which 603 candidates came out successful. Subject wise results are as follows:

\begin{tabular}{llccc}
\hline SI No. & Subject & Appeared & Passed & \% of Pass \\
\hline 1. & Medicine & 1192 & 209 & 17.53 \\
2. & Surgery & 666 & 146 & 21.92 \\
3. & Paediatrics & 395 & 47 & 11.90 \\
4. & Obst. And Gynae & 796 & 98 & 12.31 \\
5. & Otolaryngology & 110 & 13 & 11.82 \\
6. & Ophthalmology & 86 & 11 & 12.79 \\
7. & Psychiatry & 13 & 1 & 7.69 \\
8. & Anaesthesiology & 86 & 8 & 9.30 \\
9 & Radiology & 75 & 17 & 22.67 \\
10 & Radiotherapy & 30 & 1 & 3.33 \\
11 & Dermatology and Venereology & 91 & 2 & 2.20 \\
12 & Physical Medicine \& Rehabilitation & 29 & 7 & 24.14 \\
13 & Dentistry & 190 & 29 & 15.26 \\
14 & Family Medicine & 0 & 0 & 0.00 \\
15 & Haematology & 26 & 10 & 38.46 \\
16 & Microbiology & 12 & 1 & 8.33 \\
17 & Histopathology & 14 & 2 & 14.29 \\
18 & Transfusion Medicine & 2 & 1 & 50.00 \\
\hline & Grand Total & $\mathbf{3 8 1 3}$ & $\mathbf{6 0 3}$ & $\mathbf{1 5 . 8 1}$ \\
\hline
\end{tabular}

The following candidates satisfied the Board of Examiners and were declared to have passed the FCPS examination held in July, 2011 subject to confirmation by the council of Bangladesh College of Physicians and Surgeons.

\begin{tabular}{llll}
\hline Roll No. & Name & From where graduated & Subject \\
\hline $018-8701$ & Dr. Shahana Zaman & Sir Salimullah Medical College, Dhaka & Cardiology \\
$018-8702$ & Dr. Shaila Nabi & Sir Salimullah Medical College, Dhaka & Cardiology \\
$018-8706$ & Dr. Abu Daud Md Shariful Islam & Chittagong Medical College, Chittagong & Paediatric Surgery \\
$018-8708$ & Dr. Mohammed Rashedul Islam & Chittagong Medical College, Chittagong & Plastic and Recon. Surgery \\
$079-7002$ & Dr. Shahriar Ahmed & Rajshahi Medical College, Rajshahi & Anaesthesiology \\
$079-7003$ & Dr. Habibul Hai Masud & Mymensingh Medical College, Mymensingh & Anaesthesiology \\
$079-7005$ & Dr. Nepal Chandra Saha & Sher-E-Bangla Medical College, Barisal & Anaesthesiology \\
$079-7007$ & Dr. Mohammed Mostafizur Rahman & Sir Salimullah Medical College, Dhaka & Anaesthesiology \\
$079-7009$ & Dr. Lubna Naznin & Dhaka Medical College, Dhaka & Biochemistry \\
$079-7013$ & Dr. Md. Shafiqul Alam & Dhaka Dental College, Dhaka & Conservative Dentistry \\
$079-7014$ & Dr. Md. Ismail Hossain & Rajshahi Medical College, Rajshahi & Conservative Dentistry \\
$079-7015$ & Dr. ATM Rezaul Karim & Rajshahi Medical College, Rajshahi & Dermatology and Venereology \\
\hline
\end{tabular}




\begin{tabular}{|c|c|c|c|}
\hline Roll No. & Name & From where graduated & Subject \\
\hline 079-7020 & Dr. Mohammad Abdullah-Hel Kafi & Dhaka Medical College, Dhaka & Dermatology and Venereology \\
\hline 079-7032 & Dr. Kismat Ara Islam & Dhaka Medical College, Dhaka & Dermatology and Venereology \\
\hline 079-7039 & Dr. Abu Reza Sayem Ahamed & Sher-E-Bangla Medical College, Barisal & Dermatology and Venereology \\
\hline 079-7042 & Dr. Mohammad Niamat Alahi & Dhaka Medical College, Dhaka & Dermatology and Venereology \\
\hline $079-4043$ & Dr. Mst. Moriom Nesa & Rajshahi Medical College, Rajshahi & Dermatology and Venereology \\
\hline 079-7050 & Dr. Farida Arjuman & Chittagong Medical College, Chittagong & Histopathology \\
\hline 079-7059 & Dr. Syed Abul Foez & Mymensingh Medical College, Mymensingh & Medicine \\
\hline 079-7060 & Dr. Sonia Nasreen Ahmad & Bangladesh Medical College, Dhaka & Medicine \\
\hline 079-7063 & Dr. Mohammad Reazul Karim & Sir Salimullah Medical College, Dhaka & Medicine \\
\hline 079-7073 & Dr. Gobinda Chandra Banik & Sir Salimullah Medical College, Dhaka & Medicine \\
\hline 079-7095 & Dr. Md. Amir Hossain & Chittagong Medical College, Chittagong & Medicine \\
\hline 079-7099 & Dr. Prabir Mohan Basak & Rajshahi Medical College, Rajshahi & Medicine \\
\hline 079-7100 & Dr. Md Faridul Islam & Chittagong Medical College, Chittagong & Medicine \\
\hline 079-7106 & Dr. Hasna Fahmima Haque & Rangpur Medical College, Rangpur & Medicine \\
\hline 079-7107 & Dr. Abed Hussain Khan & Jahurul Islam Medical College, Bajitpur & Medicine \\
\hline 079-7110 & Dr. Ashfaque Ahmmed & Rangpur Medical College, Rangpur & Medicine \\
\hline 079-7111 & Dr. A S M Rezaul Karim & Rajshahi Medical College, Rajshahi & Medicine \\
\hline 079-7118 & Dr. Reaz Mahmud Huda & Mymensing Medical College, Mymensing & Medicine \\
\hline 079-7142 & Dr. Mohammad Anwarul Bari & Dhaka Medical College, Dhaka & Medicine \\
\hline $079-7167$ & Dr. Mohammad Rafiqul Islam & Mymensing Medical College, Mymensing & Medicine \\
\hline 079-7174 & Dr. Mohiuddin Ahmed & Sir Salimullah Medical College, Dhaka & Medicine \\
\hline $079-7175$ & Dr. Mohammad Ala Uddin & Dinajpur Medical College, Dinajpur & Medicine \\
\hline $079-7178$ & Dr. Kazi Mohammad Abrar Hasan & Dhaka Medical College, Dhaka & Medicine \\
\hline 079-7182 & Dr. Aminur Rahman & MAG Osmani Medical College, Sylhet & Medicine \\
\hline 079-7183 & Dr. Ranjon Kumer Roy & Rangpur Medical College, Rangpur & Medicine \\
\hline 079-7192 & Dr. Mohammad Shaheen Sikder & Dhaka Medical College, Dhaka & Medicine \\
\hline 079-7195 & Dr. Mohammad Mosharof Hossain & Faridpur Medical College, Faridpur & Medicine \\
\hline 079-7202 & Dr. Mohammad Syedul Islam & MAG Osmani Medical College, Sylhet & Medicine \\
\hline 079-7207 & Dr. Sheikh Khairul Kabir & Dinajpur Medical College, Dinajpur & Medicine \\
\hline 079-7209 & Dr. Mohammad Iqbal Hossain & Dhaka Medical College, Dhaka & Medicine \\
\hline 079-7211 & Dr. Al-Muksit Md. Taufiquer Rahman & Rangpur Medical College, Rangpur & Medicine \\
\hline $079-7223$ & Dr. Md. Nuruzzaman & Rangpur Medical College, Rangpur & Medicine \\
\hline $079-7224$ & Dr. Md. Shahidul Islam & Shahid Ziaur Rahman Medical College, Bogra & Medicine \\
\hline 079-7228 & Dr. Md Aynul Islam & Rangpur Medical College, Rangpur & Medicine \\
\hline $079-7229$ & Dr. Md Abul Kalam Azad & Rajshahi Medical College, Rajshahi & Medicine \\
\hline 079-7239 & Dr. Tapash Saha & Dhaka Medical College, Dhaka & Medicine \\
\hline $079-7253$ & Dr. Ummay Fatema Khatun & Chittagong Medical College, Chittagong & Medicine \\
\hline $079-7260$ & Dr. Mohammad Zaynal Abedin & Comilla Medical College, Comilla & Medicine \\
\hline 079-7266 & Dr. Mohammed Gias Uddin & Chittagong Medical College, Chittagong & Medicine \\
\hline 079-7269 & Dr. Atia Saeed & Mymensing Medical College, Mymensing & Medicine \\
\hline 079-7272 & Dr. Debasish Kumar Saha & Dhaka Medical College, Dhaka & Medicine \\
\hline $079-7274$ & Dr. Md Abu Bakar Siddique & Mymensing Medical College, Mymensing & Medicine \\
\hline $079-7278$ & Dr. Mohammad Murad Hossain & Dhaka Medical College, Dhaka & Medicine \\
\hline 079-7279 & Dr. Sabrina Yesmin & Sir Salimullah Medical College, Dhaka & Medicine \\
\hline 079-7282 & Dr. Goutam Kumar Acherjya & Sher-E-Bangla Medical College, Barisal & Medicine \\
\hline $079-7287$ & Dr. Muhammad Shaista Ali Khan & Rangpur Medical College, Rangpur & Medicine \\
\hline $079-7288$ & Dr. Muhammad Anwarul Kabir & Dhaka Medical College, Dhaka & Medicine \\
\hline
\end{tabular}


College News and CPD Programme

\begin{tabular}{|c|c|c|c|}
\hline Roll No. & Name & From where graduated & Subject \\
\hline 079-7289 & Dr. Abdur Razzaque & Rangpur Medical College, Rangpur & Medicine \\
\hline 079-7295 & Dr. Mohammad Abul Kalam Azad & Sir Salimullah Medical College, Dhaka & Medicine \\
\hline 079-7300 & Dr. Md. Abul Kalam Azad & Dhaka Medical College, Dhaka & Medicine \\
\hline 079-7312 & Dr. Mohammed Abdur Rahim & MAG Osmani Medical College, Sylhet & Medicine \\
\hline 079-7316 & Dr. Mohammad Ahsanul Haque & Rangpur Medical College, Rangpur & Medicine \\
\hline 079-7317 & Dr. Abu noim Muhammad Saifullah & Mymensing Medical College, Mymensing & Medicine \\
\hline 079-7324 & Dr. Mohammad Enamul Karim & Sir Salimullah Medical College, Dhaka & Medicine \\
\hline 079-7336 & Dr. Md. Sarwar Khan & Sir Salimullah Medical College, Dhaka & Medicine \\
\hline 079-7337 & Dr. Suvash Chandra Vadury & Sir Salimullah Medical College, Dhaka & Medicine \\
\hline 079-7349 & Dr. Mohammad Ashif Rahman & Chittagong Medical College, Chittagong & Medicine \\
\hline $079-7358$ & Dr. Mohammad Jahangir Alam Sarker & Faridpur Medical College, Faridpur & Medicine \\
\hline $079-7365$ & Dr. Md Rafiqul Islam & Rajshahi Medical College, Rajshahi & Medicine \\
\hline 079-7376 & Dr. Md. Yasir Arafat & Chittagong Medical College, Chittagong & Medicine \\
\hline 079-7387 & Dr. Syeda Fahmida Hossain & Dhaka Medical College, Dhaka & Medicine \\
\hline $079-7388$ & Dr. Rama Biswas & Sir Salimullah Medical College, Dhaka & Medicine \\
\hline 079-7389 & Dr. Umma Salma & Dhaka Medical College, Dhaka & Medicine \\
\hline 079-7393 & Dr. Jishu Deb Nath & Chittagong Medical College, Chittagong & Medicine \\
\hline 079-7405 & Dr. Khaleda Akter & Chittagong Medical College, Chittagong & Obst and Gynae \\
\hline $079-7452$ & Dr. Nayeema Begum & Sher-E-Bangla Medical College, Barisal & Obst and Gynae \\
\hline 079-7464 & Dr. Mowshumi Jahan & Sir Salimullah Medical College, Dhaka & Obst and Gynae \\
\hline 079-7476 & Dr. A. Wahid Uddin & Rajshahi Medical College, Rajshahi & Obst and Gynae \\
\hline 079-7479 & Dr. Raihana Amin & Mymensing Medical College, Mymensing & Obst and Gynae \\
\hline $079-7506$ & Dr. Mina Rani Debi & Rangpur Medical College, Rangpur & Obst and Gynae \\
\hline 079-7534 & Dr. Afroza & Community Based Medical College, Mymensing & Obst and Gynae \\
\hline 079-7554 & Dr. Ayesha Beg & Dhaka Medical College, Dhaka & Obst and Gynae \\
\hline 079-7568 & Dr. Fatheha Ferdous & Comilla Medical College, Comilla & Obst and Gynae \\
\hline 079-7570 & Dr. Housneara begum & Mymensing Medical College, Mymensing & Obst and Gynae \\
\hline 079-7580 & Dr. Parveen Akter & Chittagong Medical College, Chittagong & Obst and Gynae \\
\hline 079-7588 & Dr. Rowshan Ara Begum & Dhaka Medical College, Dhaka & Obst and Gynae \\
\hline 079-7589 & Dr. Farzana Rashid & Mymensing Medical College, Mymensing & Obst and Gynae \\
\hline 079-7601 & Dr. Simla Aftab Shaon & Mymensing Medical College, Mymensing & Obst and Gynae \\
\hline 079-7604 & Dr. Ebana Begum & MAG Osmani Medical College, Sylhet & Obst and Gynae \\
\hline 079-7611 & Dr. Farzana Islam & Rangpur Medical College, Rangpur & Obst and Gynae \\
\hline 079-7612 & Dr. Lubna Yasmin & Sir Salimullah Medical College, Dhaka & Obst and Gynae \\
\hline $079-7613$ & Dr. Sabrina Ahmed & Sir Salimullah Medical College, Dhaka & Obst and Gynae \\
\hline 079-7648 & Dr. Khandaker Shehneela Tasmin & Mymensing Medical College, Mymensing & Obst and Gynae \\
\hline 079-7650 & Dr. Kazi Mobina Akhtar & Rajshahi Medical College, Rajshahi & Obst and Gynae \\
\hline $079-7663$ & Dr. Tanzima Siddiquah & Dhaka Medical College, Dhaka & Obst and Gynae \\
\hline 079-7690 & Dr. Sabita Dhar & MAG Osmani Medical College, Sylhet & Obst and Gynae \\
\hline 079-7754 & Dr. Monowara Begum & Rajshahi Medical College, Rajshahi & Obst and Gynae \\
\hline 079-7781 & Dr. Sharmin Siddika & North-East Medical College, Sylhet & Obst and Gynae \\
\hline $079-7825$ & Dr. Munjura Akhtar & Sir Salimullah Medical College, Dhaka & Obst and Gynae \\
\hline 079-7830 & Dr. Afroza Siddiqua & Rangpur Medical College, Rangpur & Obst and Gynae \\
\hline 079-7845 & Dr. Alpana Adhikary & Sher-E-Bangla Medical College, Barisal & Obst and Gynae \\
\hline 079-7847 & Dr. Mamata Manjari & Sir Salimullah Medical College, Dhaka & Obst and Gynae \\
\hline 079-7849 & Dr. Kanchan Sarker & Dhaka Medical College, Dhaka & Obst and Gynae \\
\hline $079-7851$ & Dr. Sheikh Abdul Moktad & Rangpur Medical College, Rangpur & Ophthalmology \\
\hline
\end{tabular}




\begin{tabular}{|c|c|c|c|}
\hline Roll No. & Name & From where graduated & Subject \\
\hline $079-7852$ & Dr. Farhana Yasmin & MAG Osmani Medical College, Sylhet & Ophthalmology \\
\hline $079-7855$ & Dr. Sabrina Rahmatullah & Armed Forces Medical College, Dhaka & Ophthalmology \\
\hline $079-7860$ & Dr. Md Nazrul Islam & Rajshahi Medical College, Rajshahi & Ophthalmology \\
\hline $079-7863$ & Dr. Md Abul Kalam Azad & Dhaka Medical College, Dhaka & Ophthalmology \\
\hline $079-7864$ & Dr. Md. Sibgatullah & Dhaka Medical College, Dhaka & Ophthalmology \\
\hline $079-7868$ & Dr. Asif-Ur-Rahman & Sir Salimullah Medical College, Dhaka & Ophthalmology \\
\hline 079-7869 & Dr. Mohammad Syed Jahangir Kabir & MAG Osmani Medical College, Sylhet & Ophthalmology \\
\hline $079-7876$ & Dr. Md. Atiquzzaman & Rajshahi Medical College, Rajshahi & Ophthalmology \\
\hline 079-7877 & Dr. Maria Akhtar Dalia & Rajshahi Medical College, Rajshahi & Ophthalmology \\
\hline $079-7878$ & Dr. Bipul Chandra Mondal & Rangpur Medical College, Rangpur & Ophthalmology \\
\hline $079-7881$ & Dr. Dilara Khatun & Rajshahi Medical College, Rajshahi & Ophthalmology \\
\hline $079-7883$ & Dr. Md. Nazrul islam & Sir Salimullah Medical College, Dhaka & Ophthalmology \\
\hline $079-7884$ & Dr. Tawfiq Uddin Ahmed & Khulna Medical College, Khulna & Ophthalmology \\
\hline $079-7887$ & Dr. Mohammad Mazaharul Islam & Sher-E-Bangla Medical College, Barisal & Ophthalmology \\
\hline 079-7891 & Dr. Mohammad Wahidul Islam & Dhaka Dental College, Dhaka & Oral and Maxillofacial Surgery \\
\hline 079-7899 & Dr. Md Apel Mamood & Dhaka Dental College, Dhaka & Oral and Maxillofacial Surgery \\
\hline 079-7904 & Dr. Hasan Mohammad Rizvi & Dhaka Dental College, Dhaka & Orthodontics and Dentofacial Orthopaedics \\
\hline 079-7906 & Dr. Md Golam Rubby & Dhaka Dental College, Dhaka & Orthodontics and Dentofacial Orthopaedics \\
\hline 079-7908 & Dr. Md. Anisur rahman & Dhaka Dental College, Dhaka & Orthodontics and Dentofacial Orthopaedics \\
\hline $079-7910$ & Dr. Md Liakat Ali Hyder & Dhaka Dental College, Dhaka & Orthodontics and Dentofacial Orthopaedics \\
\hline 079-7913 & Dr. Sarder Mohammad Golam Rabbani & Chittagong Medical College, Chittagong Otolaryngology & \\
\hline 079-7921 & Dr. Md Aktar Kamal & Chittagong Medical College, Chittagong & Otolaryngology \\
\hline 079-7924 & Dr. Md Mostafizur Rahman & Sher-E-Bangla Medical College, Barisal & Otolaryngology \\
\hline $079-7925$ & Dr. Bicithra Kumar Dey & Comilla Medical College, Comilla & Otolaryngology \\
\hline $079-7928$ & Dr. A K M Asaduzzaman & Sher-E-Bangla Medical College, Barisal & Otolaryngology \\
\hline 079-7932 & Dr. muhammad ali azad & Sir Salimullah Medical College, Dhaka & Otolaryngology \\
\hline $079-7938$ & Dr. Kona Chowdhury & Dhaka Medical College, Dhaka & Paediatrics \\
\hline 079-7943 & Dr. Mahmuda Zaman & Dhaka Medical College, Dhaka & Paediatrics \\
\hline 079-7944 & Dr. Most. Umme habiba begum & Rangpur Medical College, Rangpur & Paediatrics \\
\hline $079-7945$ & Dr. Most. Ummekulsum begum & Dinajpur Medical College, Dinajpur & Paediatrics \\
\hline 079-7949 & Dr. Ruma Parvin & Jahurul Islam Medical College, Bajitpur & Paediatrics \\
\hline $079-7975$ & Dr. Banita Mistry & Dhaka Medical College, Dhaka & Paediatrics \\
\hline $079-7976$ & Dr. Mahbuba Sultana & Sir Salimullah Medical College, Dhaka & Paediatrics \\
\hline $079-7983$ & Dr. Be-Nazir Ahmmad & Rajshahi Medical College, Rajshahi & Paediatrics \\
\hline $079-7998$ & Dr. Farah Diba & Rajshahi Medical College, Rajshahi & Paediatrics \\
\hline $079-8003$ & Dr. Rumy Tabrez Hyder & Dhaka Medical College, Dhaka & Paediatrics \\
\hline $079-8005$ & Dr. Md Jahidur Rahman & Dhaka Medical College, Dhaka & Paediatrics \\
\hline $079-8018$ & Dr. Md Jashim Uddin & Sher-E-Bangla Medical College, Barisal & Paediatrics \\
\hline $079-8021$ & Dr. Ashis Kumar Halder & Sher-E-Bangla Medical College, Barisal & Paediatrics \\
\hline $079-8026$ & Dr. Nusrat Nahar & Rangpur Medical College, Rangpur & Paediatrics \\
\hline $079-8036$ & Dr. Monika Mazumder & Rangpur Medical College, Rangpur & Paediatrics \\
\hline $079-8078$ & Dr. Md. Khairuzzaman & Rangpur Medical College, Rangpur & Paediatrics \\
\hline 079-8093 & Dr. Farzana Afrooz & Sir Salimullah Medical College, Dhaka & Paediatrics \\
\hline 079-8094 & Dr. Tasnima Ahmed & Mymensing Medical College, Mymensing & Paediatrics \\
\hline 079-8099 & Dr. Farhana Rahman & Dhaka Medical College, Dhaka & Paediatrics \\
\hline $079-8103$ & Dr. Md. Imamur rashid & Mymensing Medical College, Mymensing & Physical Medicine \& Rehabilitation \\
\hline
\end{tabular}


College News and CPD Programme

\begin{tabular}{|c|c|c|c|}
\hline Roll No. & Name & From where graduated & Subject \\
\hline 079-8104 & Dr. Md. Habibur rahman & Faridpur Medical College, Faridpur & Physical Medicine \& Rehabilitation \\
\hline 079-8107 & Dr. Mohammad Ilias & Chittagong Medical College, Chittagong & Physical Medicine \& Rehabilitation \\
\hline 079-8108 & Dr. Muhammad Kamrul Hassan & Chittagong Medical College, Chittagong & Physical Medicine \& Rehabilitation \\
\hline 079-8109 & Dr. Mohammad Tariqul Islam & Chittagong Medical College, Chittagong & Physical Medicine \& Rehabilitation \\
\hline $079-8110$ & Dr. Nitai Prashad Dutta & Chittagong Medical College, Chittagong & Physical Medicine \& Rehabilitation \\
\hline $079-8113$ & Dr. Niaz Mohammad Khan & Sir Salimullah Medical College, Dhaka & Psychiatry \\
\hline $079-8114$ & Dr. Mohammad Tariqul Alam & Sher-E-Bangla Medical College, Barisal & Psychiatry \\
\hline $079-8128$ & Dr. Kazi Iftekhar Uddin Ahmed & Faridpur Medical College, Faridpur & Radiotherapy \\
\hline $079-8129$ & Dr. Tapash Mitra & Chittagong Medical College, Chittagong & Radiotherapy \\
\hline $079-8130$ & Dr. Mohammad Ali Asgar Chowdhury & Chittagong Medical College, Chittagong & Radiotherapy \\
\hline $079-8131$ & Dr. Mohammad Kamruzzaman & Chittagong Medical College, Chittagong & Radiotherapy \\
\hline $079-8163$ & Dr. Mohammad Ershad Alam & Rangpur Medical College, Rangpur & Surgery \\
\hline $079-8175$ & Dr. Md. Jahidul islam & Shahid Ziaur Rahman Medical College, Bogra & Surgery \\
\hline $079-8200$ & Dr. Md. Touhidul islam & Dhaka Medical College, Dhaka & Surgery \\
\hline 079-8202 & Dr. Md. Aziz ullah & Sir Salimullah Medical College, Dhaka & Surgery \\
\hline $079-8221$ & Dr. Haridas Saha & Mymensing Medical College, Mymensing & Surgery \\
\hline $079-8223$ & Dr. Md Shohidul Islam & Dhaka Medical College, Dhaka & Surgery \\
\hline $079-8231$ & Dr. Md Ashiqur Rahman & Chittagong Medical College, Chittagong & Surgery \\
\hline $079-8266$ & Dr. Md. Shamim hossain & Rangpur Medical College, Rangpur & Surgery \\
\hline $079-8274$ & Dr. Khanduker md. Shohel uillah & Rangpur Medical College, Rangpur & Surgery \\
\hline $079-8296$ & Dr. Shahidul Huq & Jahurul Islam Medical College, Bajitpur & Surgery \\
\hline $079-8298$ & Dr. Mohammed Rashed Uz Zaman & Comilla Medical College, Comilla & Surgery \\
\hline $079-8308$ & Dr. Omar Siddiqui & Sir Salimullah Medical College, Dhaka & Surgery \\
\hline $079-8317$ & Dr. Md Jamal-E-Rabby & Rangpur Medical College, Rangpur & Surgery \\
\hline $079-8328$ & Dr. Md. Rayhanur rahman & Rajshahi Medical College, Rajshahi & Surgery \\
\hline 079-8339 & Dr. Md. Atikul ahsan & Sir Salimullah Medical College, Dhaka & Surgery \\
\hline $079-8342$ & Dr. Md. Golam kabir & Dhaka Medical College, Dhaka & Surgery \\
\hline $079-8352$ & Dr. Md. Humayun kabir & Sir Salimullah Medical College, Dhaka & Surgery \\
\hline $079-8354$ & Dr. Nilufar Shabnam & Bangladesh Medical College, Dhaka & Surgery \\
\hline $079-8357$ & Dr. Ajmal Quader Chowdhury & Bangladesh Medical College, Dhaka & Surgery \\
\hline
\end{tabular}

The following candidates satisfied the Board of Examiners and are declared to have passed the Preli - FCPS - II Exam held in July 2011 subject to confirmation by the council of Bangladesh College of Physicians and Surgeons

\begin{tabular}{llll}
\hline Roll No. & Name & From where graduated & Subject \\
\hline 013-8409 & Dr. Subrata Prokash Kar & Sher-E-Bangla Medical College, Barisal & Preli - Medicine \\
013-8434 & Dr. Mahmuda Khatun & Z.H. Sikder Women's Medical College, Dhaka & Preli - Paediatrics \\
013-8435 & Dr. Nishat Jahan & Rajshahi Medical College, Rajshahi & Preli - Paediatrics \\
013-8442 & Dr. Kazi Imran Ahmed & Sher-E-Bangla Medical College, Barisal & Preli - Surgery \\
013-8449 & Dr. Md. Towhid belal & Sir Salimullah Medical College, Dhaka & Preli - Surgery \\
$013-8463$ & Dr. Md Shariful Islam & Shahid Ziaur Rahman Medical College, Bogra & Preli - Surgery \\
\hline
\end{tabular}


The following candidates satisfied the Board of Examiners and are declared to have passed the MCPS Examination held in July 2011 subject to confirmation by the council of Bangladesh College of Physicians and Surgeons.

\begin{tabular}{|c|c|c|c|}
\hline Roll No. & Name & From where graduated & Subject \\
\hline $079-9002$ & Dr. Rashed Minhaz Siddiquei & MAG Osmani Medical College, Sylhet & Anaesthesiology \\
\hline $079-9006$ & Dr. Md. Kutub Uddin Khan & Sher-E-Bangla Medical College, Barisal & Anaesthesiology \\
\hline $079-9007$ & Dr. Sattayajit Dutta & Sher-E-Bangla Medical College, Barisal & Anaesthesiology \\
\hline $079-9010$ & Dr. Monirul Islam & Rajshahi Medical College & Anaesthesiology \\
\hline $079-9020$ & Dr. Md. Tarikul Hasan & Shahid Ziaur Rahman Medical College, Bogra & Anaesthesiology \\
\hline $079-9032$ & Dr. Mohammad Elias Hossain & Sir Salimullah Medical College, Dhaka & Clinical Pathology \\
\hline $079-9034$ & Dr. Rubaiyat-E-Mortaz & Bangladesh Medical College, Dhaka & Clinical Pathology \\
\hline $079-9035$ & Dr. Most. Sarmin Sultana & Rajshahi Medical College & Clinical Pathology \\
\hline $079-9036$ & Dr. Md. Saiful Islam & Sher-E-Bangla Medical College, Barisal & Clinical Pathology \\
\hline $079-9045$ & Dr. ANM Nazmul Hoque & Dhaka Dental College, Dhaka & Dental Surgery \\
\hline $079-9048$ & Dr. Mohd. Nurul Alam & Dhaka Medical College, Dhaka & Dermatology and Venereology \\
\hline $079-9050$ & Dr. Laila Afroz & Sir Salimullah Medical College, Dhaka & Dermatology and Venereology \\
\hline $079-9060$ & Dr. Md. Delwar Hossain & Rangpur Medical College & Family Medicine \\
\hline $079-9064$ & Dr. Sanchay Kumar Biswas & Rajshahi Medical College, Rajshahi & Family Medicine \\
\hline $079-9068$ & Dr. Khan Shakil Ahmed & Jahurul Islam Medical College, Bajitpur & Forensic Medicine \\
\hline $079-9069$ & Dr. Md. Rezaul Karim & Armed Forces Medical College, Dhaka & Forensic Medicine \\
\hline 079-9071 & Dr. A.K.M. Shafiuzzaman & Rangpur Medical Colllege, Rangpur & Forensic Medicine \\
\hline $079-9072$ & Dr. Mohammed Mazharul Islam & Community Based Medical College, Mymensing & Forensic Medicine \\
\hline $079-9081$ & Dr. Hiranmoy Dutta & Chittagong Medical College, Chittagong & Medicine \\
\hline 079-9098 & Dr. Rajib Ghosh & Dhaka Medical College, Dhaka & Medicine \\
\hline $079-9114$ & Dr. Mohammed Nasir Uddin & Chittagong Medical College, Chittagong & Medicine \\
\hline $079-9130$ & Dr. Rabiul Alam Md. Erfan Uddin & Dhaka Medical College, Dhaka & Medicine \\
\hline $079-9166$ & Dr. Md. Ahsan Ullah Rumi & Sir Salimullah Medical College, Dhaka & Medicine \\
\hline $079-9186$ & Dr. Shahana Sultana & Sher-E-Bangla Medical College, Barisal & Obst and Gynae \\
\hline $079-9194$ & Dr. Seema Rani Dabee & Sir Salimullah Medical College, Dhaka & Obst and Gynae \\
\hline 079-9197 & Dr. Amina Khatunn & Rajshahi Medical College, Rajshahi & Obst and Gynae \\
\hline $079-9223$ & Dr. Ayesha Aktar & Sher-E-Bangla Medical College, Barisal & Obst and Gynae \\
\hline $079-9237$ & Dr. Runa Laila & MAG Osmani Medical College, Sylhet & Obst and Gynae \\
\hline $079-9256$ & Dr. Romena Akter & Sher-E-Bangla Medical College, Barisal & Obst and Gynae \\
\hline $079-9259$ & Dr. Mahabuba Ahmed & Chittagong Medical College, Chittagong & Obst and Gynae \\
\hline $079-9261$ & Dr. Sharmin Hossain & Medical College for Women, Uttara, Dhaka & Obst and Gynae \\
\hline $079-9263$ & Dr. Ayesha Nazneen & Chittagong Medical College, Chittagong & Obst and Gynae \\
\hline $079-9267$ & Dr. Kamrunnahar & Rangpur Medical Colllege, Rangpur & Obst and Gynae \\
\hline $079-9270$ & Dr. Shamima Afroz & Sher-E-Bangla Medical College, Barisal & Obst and Gynae \\
\hline $079-9273$ & Dr. Roksana Begum & Sir Salimullah Medical College, Dhaka & Obst and Gynae \\
\hline $079-9274$ & Dr. Rawshan Ara Begum & Rangpur Medical College, Rangpur & Obst and Gynae \\
\hline $079-9281$ & Dr. Shamima Hasrin & Sir Salimullah Medical College, Dhaka & Obst and Gynae \\
\hline $079-9284$ & Dr. Fahmida Monir & Chittagong Medical College, Chittagong & Obst and Gynae \\
\hline $079-9287$ & Dr. Minara Sidker & Faridpur Medical College, Faridpur & Obst and Gynae \\
\hline 079-9295 & Dr. Fatema Khatyun & Sir Salumullah Medical College, Dhaka & Obst and Gynae \\
\hline 079-9297 & Dr. Anumanara & Rajshahi Medical College, Rajshahi & Obst and Gynae \\
\hline $079-9307$ & Dr. Aysha Siddiquea & Mymensingh Medical College, Mymensing & Obst and Gynae \\
\hline 079-9309 & Dr. Eliza Shireen & Mymensingh Medical College, Mymensing & Obst and Gynae \\
\hline 079-9314 & Dr. Sazia Parveen & Bangladesh Medical College, Dhaka & Obst and Gynae \\
\hline $079-9316$ & Dr. AKM Rezaul Islam & Rangpur Medical College, Rangpr & Ophthalmology \\
\hline 079-9317 & Dr. Uhammad Nazmul Haque & Chittagong Medical College, Chittagong & Ophthalmology \\
\hline
\end{tabular}




\begin{tabular}{llll}
\hline Roll No. & Name & From where graduated & Subject \\
\hline $079-9319$ & Dr. Md. Mominul Islam & Holy Family Red Crescent Medical College & Ophthalmology \\
$079-9327$ & Dr. Sk Mohammad Murad & Jahrul Islam Medical College, Bajitpur & Ophthalmology \\
$079-9328$ & Dr. Mohmmedul Haque & Chittagong Medical College, Chittagong & Ophthalmology \\
$079-9332$ & Dr. Billal Hossain & Dhaka Medical College, Dhaka & Ophthalmology \\
$079-9333$ & Dr. S.M. Shafiul Bari Rasel & Dhaka Medical College, Dhaka & Ophthalmology \\
$079-9334$ & Dr. Mafruha Afrin & Chittagong Medical College, Chittagong & Ophthalmology \\
$079-9348$ & Dr. Mohammad Shaharior & MAG Osmani Medicla College, Sylhet & Otolaryngology \\
$079-9350$ & Dr. Md. Zakir Hossain & Rangpur Medical College, Rangpur & Otolaryngology \\
$079-9351$ & Dr. Md. Nesar Uddin & Dhaka National Medical College, Dhaka & Otolaryngology \\
$079-9353$ & Dr. Md. Mahbubul Alam & Rangpur Medical College, Rangpur & Otolaryngology \\
$079-9369$ & Dr. Kisoar Farhana Keya & Jahurul Islam Medical College, Bajitpur & Paediatrics \\
$079-9385$ & Dr. Shafquat Waheed & Dhaka Medical College, Dhaka & Psychiatry \\
$079-9387$ & Dr. Khandker Anjumanara & Rangpur Medical College, Ragpur & Psychiatry \\
$079-9388$ & Dr. Md. Faruk Hossain & Mymensingh Medical College, Mymeningh & Psychiatry \\
$079-9394$ & Dr. A.A.M. Abu Taher & Chittagong Medical College, Chittagong & Radiotherapy \\
$079-9436$ & Dr. Md. Amir Hossain & Rangpur Medical College, Rangpur & Surgery \\
\hline
\end{tabular}

\section{CONTINUING PROFESSIONAL DEVELOPMENT (CPD) PROGRAMME}

Continuing Professional development (CPD) is a multi professional educational activity that helps professional to keep their knowledge and skills upto date in response to the changing demands of the society and professions.

According to the suggestions given by the fellows, CPD committee of BCPS has decided to arrange the programme in different institutions instead of BCPS campus and one CPD day per year.

Accordingly, we have organized 3 CPD programmes and one CPD day in BCPS. The new committee of CPD organized the $4^{\text {th }}$ programme at BSMMU on $25^{\text {th }}$ September, 2011. More than 300 postgraduate students and fellows attended the session.

\begin{tabular}{l|l|l|l|l}
\hline Date & Time & Topic & Speaker & Chairperson/ Moderator \\
\hline $25-09-2011$ & $\begin{array}{l}12: 00 \mathrm{pm} \text { to } \\
12: 30 \mathrm{pm}\end{array}$ & $\begin{array}{l}\text { Practice guide line for the } \\
\text { management of chronic } \\
\text { hepatitis B. }\end{array}$ & $\begin{array}{l}\text { Dr. Md. Shahinul Alam } \\
\text { Associate Professor of } \\
\text { Hepatology, } \\
\text { BSMMU }\end{array}$ & $\begin{array}{l}\text { Professor Salimur Rahman } \\
\text { Professor of Hepatology, } \\
\text { BSMMU }\end{array}$ \\
\hline $25-09-2011$ & $\begin{array}{l}12: 30 \mathrm{pm} \text { to } \\
1: 00\end{array}$ & $\begin{array}{l}\text { Role of Ultrasound in . } \\
\text { chest lesions }\end{array}$ & $\begin{array}{l}\text { Dr. Md. Delwar Hossain } \\
\text { Associate Professor and } \\
\text { Head of Radiology and } \\
\text { Imaging, NIKDU. }\end{array}$ & $\begin{array}{l}\text { Professor Md. Salahuddin Al-Azad } \\
\text { Chairman \& Professor, } \\
\text { BSMMU }\end{array}$ \\
& $\begin{array}{l}\text { BSadiology \& Imaging, } \\
\text { Dr. Mohammad Abul Kalam Azad } \\
\text { Associate Professor of Medicine, } \\
\text { BSMMU. }\end{array}$ \\
\hline
\end{tabular}

Prof.Kanak Kanti Barua,Honorary Secretary of BCPS,Prof.Nazmun Nahar.Past president of BCPS,Prof.S.A.M.Golam Kibria.Past Senior vice president and another councilor were present on that occasion.Prof.A. K.M Rafique Uddin, Chairman, CPD committee and Prof.Tahmina Begum,Member Secretary,CPD committee were also there.

\section{Prof.Tahmina Begum}

Member Secretary, CPD committee,BCPS 